\title{
Manual thrombectomy in Primary Percutaneous Coronary Intervention via the Transradial Approach: A case report
}

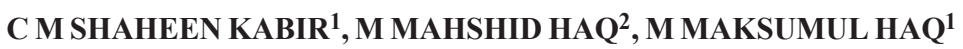 \\ ${ }^{1}$ Department of Cardiology, Ibrahim Cardiac Hospital \& Research Institute, Dhaka-1000, Bangladesh ${ }^{2}$ Internal Medicine \\ Resident, Boston University Medical Center, USA
}

Address for Correspondence: Dr. C M Shaheen Kabir, MBBS, MD (Cardiology), Assistant Professor \& Associate Consultant, Department of Cardiology, Ibrahim Cardiac Hospital \& Research Institute, Dhaka-1000, Bangladesh, e mail:

skabir67@yahoo.com, cell:+8801817578389

\begin{abstract}
::
The main goal of primary percutaneous coronary intervention (PPCI) is to achieve not only restoration of flow in the epicardial coronary artery, but also reperfusion at the level of myocardial tissue. Distal embolization is a possible complication of PPCI leading to lack of myocardial reperfusion and microvascular injury. Thrombus aspiration during PPCI has been proposed to prevent embolization. Although the use of thrombectomy devices to aspirate thrombus during primary PCI is not used routinely, there is growing evidence suggesting the use of thrombus aspiration catheters during PCI. We are reporting the case of a 53-year-old diabetic, hypertensive woman presenting with acute infero-posterior ST elevation myocardial infarction (STEMI) with right ventricular infarction. She underwent diagnostic coronary angiogram through transradial approach which revealed total occlusion of mid right coronary artery (RCA). Multiple runs of aspiration were performed using Diver Aspiration Catheter-6F and thrombus was aspirated from RCA. After successful thrombus aspiration, dramatic improvements in both coronary flow and ST-segment resolution were achieved.
\end{abstract}

Key words: Thrombus aspiration, primary PCI, transradial approach.

Introduction:

ST-segment elevation myocardial infarction (STEMI) typically occurs due to the sudden thrombotic occlusion of a coronary artery triggered by plaque rupture or erosion. The preferred management option for STEMI is primary angioplasty, including thrombus aspiration. ${ }^{1}$ Primary Percutaneous Coronary Intervention (PPCI) has significantly reduced mortality compared to fibrnolytic therapy $^{2}$, there are still a significantly large population of patients where PPCI fails to restore optimal perfusion in the infarct related artery (IRA). This is demonstrated by failure of ST-segment resolution (STR), poor myocardial blush grade (MBG) and low TIMI flow (thrombolysis in myocardial infarction). ${ }^{3}$ Hence reperfusion of an IRA does not necessarily guarantee sufficient microvascular perfusion. One of the reasons for this insufficient perfusion is high thrombus burden (resulting in increased incidence of distal embolization). Thrombus aspiration before stent implantation in the culprit lesion has been shown to improve myocardial perfusion and clinical outcomes. ${ }^{4}$

This case report describes aspiration of a thrombus from right coronary artery (RCA) through transradial approach following STEMI in a stage 4 Chronic Kidney Disease (CKD) patient.

\section{Case Report:}

A 53 year old woman, with hypertension, diabetes mellitus and CKD stage 4 was admitted to our hospital with substernal chest pain, which had developed three hour prior to presentation. No previous cardiac history was reported. She was hemodynamically stable and her cardiovascular examination was unremarkable. An electrocardiogram (ECG) showed $\geq 0.2 \mathrm{mV}$ ST segment elevation in leads II, III, aVF and reciprocal ST depression in lead I, aVL along with $\mathrm{R}$ wave and $0.1 \mathrm{mV} \mathrm{ST}$ depression \& upright-T wave in leads V1 and V2. ST segment elevation of $0.1 \mathrm{mV}$ was also present in lead V4R. ECG findings were consistent with infero-posterior STEMI with right ventricular infarction (Fig $1 \mathrm{a} \& \mathrm{~b}$ ). The patient was given aspirin (loading dose of $300 \mathrm{mg}$ orally) \& clopidogrel (loading dose of $600 \mathrm{mg}$ orally) with intention of PPCI. Cardiac enzyme (CK-MB: $32 \mathrm{U} / \mathrm{L}$, troponin-I: $0.68 \mathrm{ng} / \mathrm{mL}$ ) was elevated. Serum creatinine level was 2.9 $\mathrm{mg} / \mathrm{dl}$ with eGFR of $16.93 \mathrm{ml} / \mathrm{min} / 1.73 \mathrm{~m}^{2}$. Two-dimensional echocardiogram revealed akinesia in the RCA territories. A diagnostic coronary angiogram (CAG) through transradial approach showed thrombotic total occlusion in the mid RCA without collateral flow (Fig 2). There was non-obstructive disease in left anterior descending and left circumflex arteries. A 6 Fr JR 3.5 guiding catheter was used to cannulate RCA. A 0.014 inch ASAHI SION blue Guidewire (ASAHI INTECH, Japan) was passed across the occlusion successfully \& TIMI 0/1 flow was achieved. After low pressure pre-dilatation with a $2.5 \times 15 \mathrm{~mm}$ Sapphire II balloon (OrbusNeich), large thrombus burden was evidence in distal RCA (Fig 3) \& thrombi were aspirated using 6 Fr DIVER CEMAX catheter (INVATEC, 

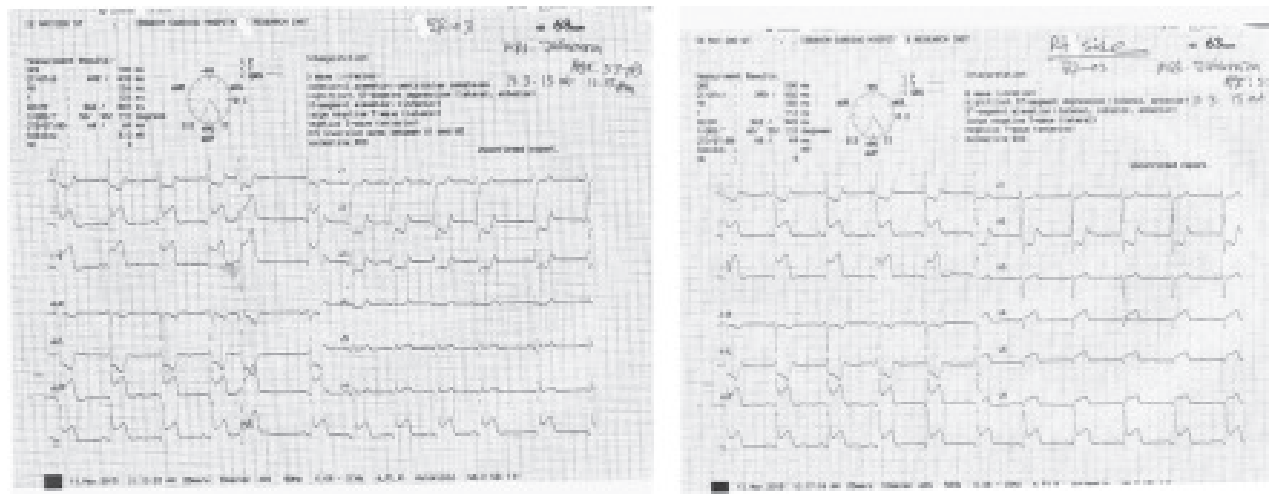

Fig.-1 a \& b: Electrocardiography showed infero-posterior STEMI with right ventricular infarction

Italy) (Fig 4 a, b \& c) followed by restoration of flow in RCA(Fig 5). A $3.0 \times 38$ mm drug eluting stent Ultimaster (TERUMO, Japan) was implanted at 16 atmosphere achieving a final TIMI flow 3 and MBG 3. ECG

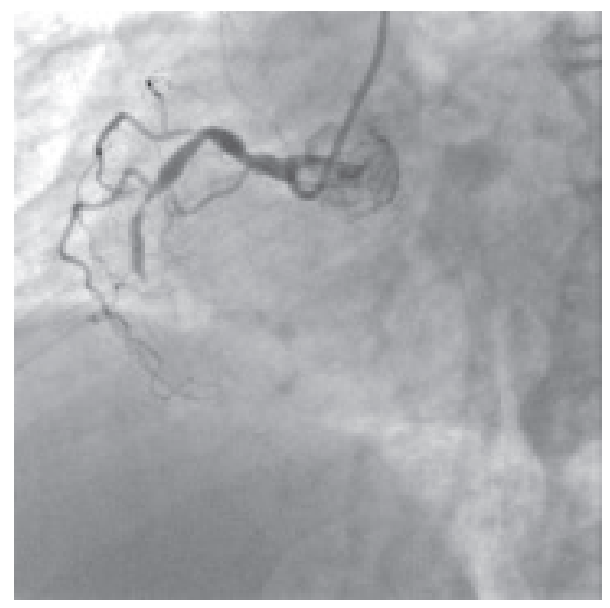

Fig.-2: $R C A$ in LAO projection showing total occlusion of the mid-RCA
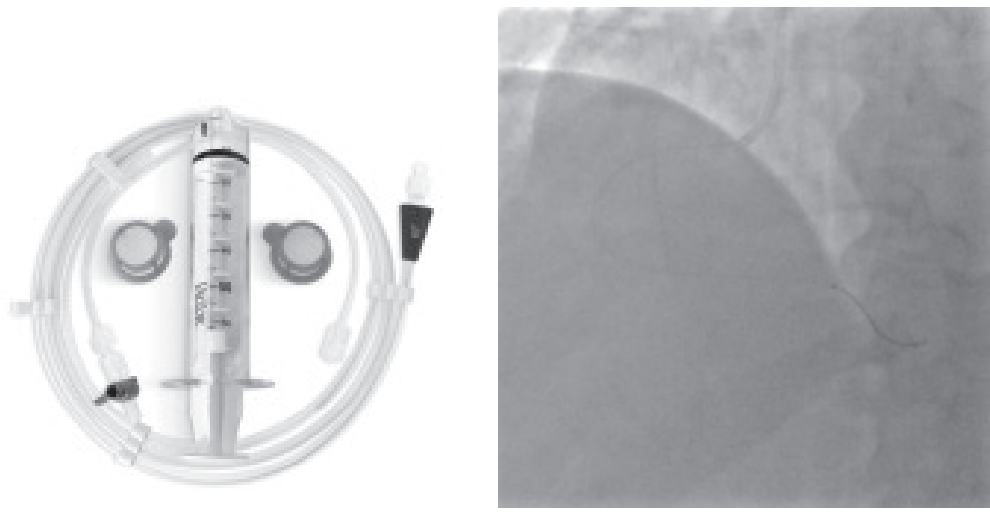

Fig.-4 a: 6 Fr DIVER CEMAX Aspiration Catheter Set used for thrombectomy via transradial approach
Fig.-4 b: Thrombus aspiration was performed in RCA multiple times demonstrated complete resolution of the elevated ST segment (Fig 6). She remained pain free and hemodynamically stable in the coronary care unit without deterioration of renal function.

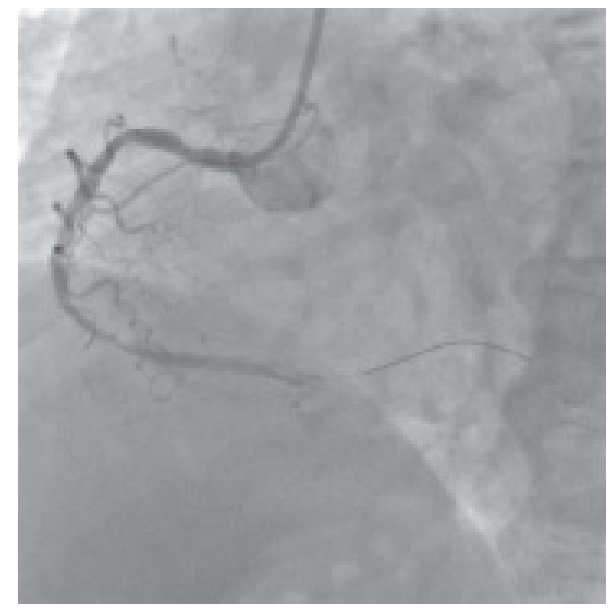

Fig.-3: $R C A$ in LAO projection showing total occlusion distally with thrombus burden after pre-dilatation

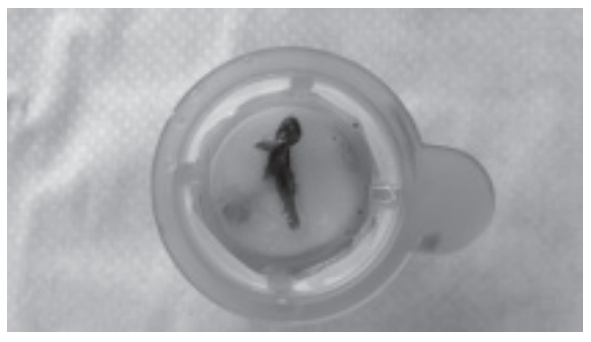

Fig.-4 c: Aspirated thrombus from $R C A$ 


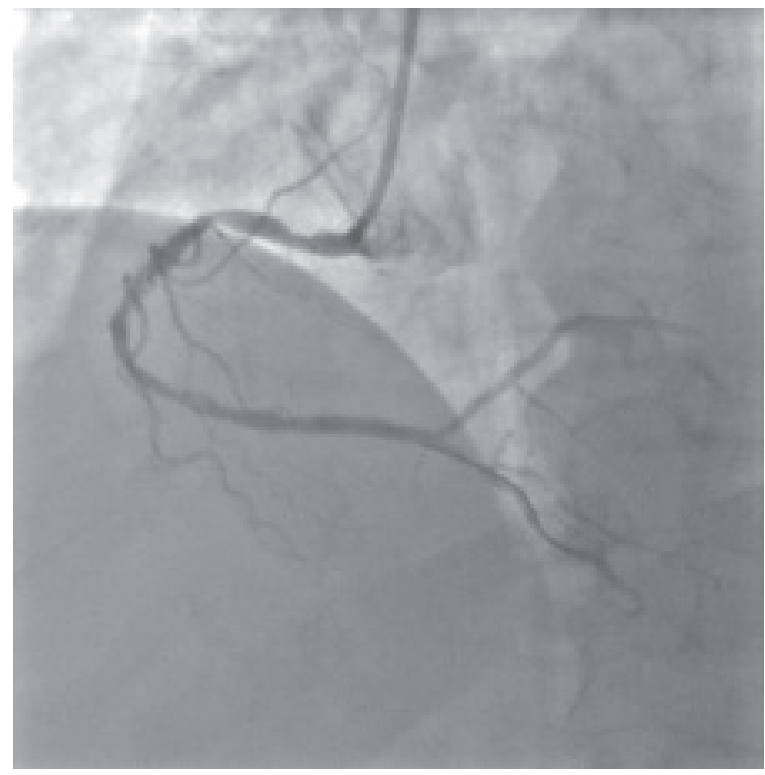

Fig.-5: LAO cranial view of RCA showed restoration of flow in RCA after thrombus aspiration

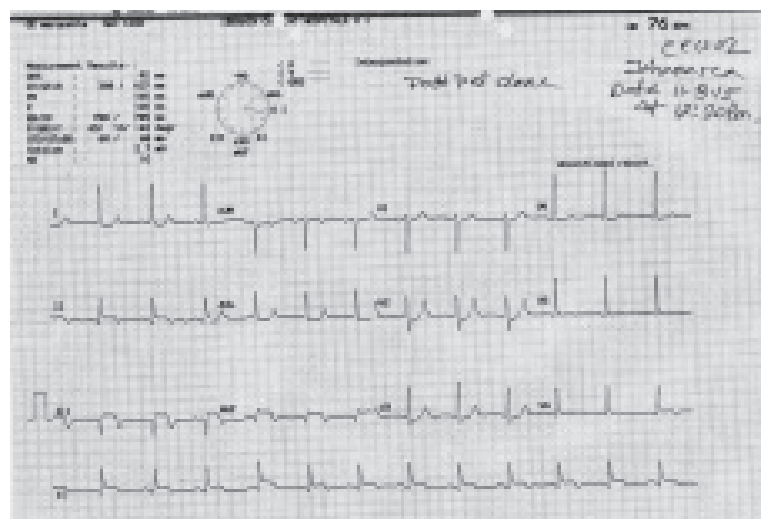

Fig.-6: Electrocardiography showed the resolution of the elevated ST segment

The patient remained free of adverse cardiac events \& was discharged home at day 4 with preserved left ventricular ejection fraction (LVEF) \& slight hypokinesia in inferior wall.

\section{Discussion:}

Acute myocardial infarction (AMI) is considered to be related primarily to the rupture or erosion of a coronary atherosclerotic plaque. This initiates intraluminal thrombus formation superimposed on the ruptured plaque which leads to total or subtotal occlusion of an epicardial coronary artery. ${ }^{5}$ PPCI has emerged as the preferred treatment of AMI if logistically feasible and has been proven to be a very effective method to obtain patency of
IRA. ${ }^{6}$ Distal embolization is a possible complication of PPCI; in these patients, a microvascular injury occurs, which finally leads to a lack of myocardial reperfusion. Thrombus aspiration during PPCI has been proposed to prevent embolization. ${ }^{7}$ Although the use of thrombectomy devices to aspirate thrombus during PPCI is currently not used routinely, there is growing evidence suggesting the use of thrombus aspiration catheters during PCI. This case highlights the effectiveness of using devices like the aspiration catheter in the setting of STEMI in restoring flow in IRA.

Manual thrombectomy devices that are currently in clinical use include the Export ${ }^{\circledR}$ Catheter (Metronic, USA), Diver (Invatec, Italy), Hunter (IHT Cordynamics, Spain), Pronto ${ }^{\circledR}$ (Vascular solutions, USA), QuickCat (Spectranetics Inc., USA) and Eliminate (Terumo) amongst others. Although these devices are similar, there are differences in the way the thrombus is extracted. ${ }^{7}$

The ESC and ACC/AHA guidelines recommend coronary artery thrombus aspiration as adjunctive therapy during PPCI for STEMI. 8,9 Thrombus aspiration is endorsed as a class IIa recommendation with the level of evidence $\mathrm{B}$ in the ACC/AHA guideline ${ }^{8}$ and with level of evidence A in the ESC guidelines. ${ }^{9}$

A number of clinical trials have assessed the use of manual thrombectomy in STEMI. One of the first randomised PPCI trials that tested a manual aspiration device was the REMEDIA (Randomised Evaluation of the Effect of Mechanical Reduction of Distal Embolisation by Thrombus Aspiration in Primary and Rescue Angioplasty) study (Diver $($ )). This study randomised 99 patients to PCI with manual aspiration or PPCI only. ${ }^{10}$ This study demonstrated that manual aspiration was associated with significantly better STR and MBG as well as reduced distal embolisation and reduced no reflow. In addition, a subgroup analysis demonstrated that thrombus aspiration was more beneficial in patients with an occluded artery and those with higher thrombus burden. ${ }^{10}$ De Luca et al. found that using manual thrombectomy in patients with anterior STEMI $(\mathrm{n}=76)$, demonstrated better post procedural MBG and better STR at 90 minutes. ${ }^{11}$ In a Bayesian meta-analysis by Mongeon et al., which included 21 trials totalling 4,299 patients (16 trials that used simple aspiration thrombectomy device), thrombectomy was shown to result in more STR and TIMI 3 flow. ${ }^{12}$

TAPAS (Thrombus Aspiration During Percutaneous Coronary Intervention in Acute Myocardial Infarction Study) was the largest trial to date in 2008. TAPAS was a single-centre randomised trial that recruited 1,071 patients 
with STEMI and randomised 535 patients to manual aspiration using the Export ${ }^{\circledR}$ catheter compared to 535 patients randomised to PPCI alone. ${ }^{13}$ Patients in the thrombectomy arm had higher MBG, higher STR and fewer pathological Q-waves. Moreover, the study showed a significant reduction in mortality with thrombus aspiration at 1 year. ${ }^{14}$

After TAPAS, a number of trials have confirmed the evidence of the benefits of manual thrombectomy. In the EXPIRA(Thrombectomy With export Catheter in InfarctRelated Artery During Primary Percutaneous Coronary Intervention) trial, 175 patients were recruited with MBG and STR as primary end points. This trial was the first study to assess thrombectomy use with microvascular obstruction as a primary end-point. MACE (Major Adverse Cardiac Events) at 9 months was the secondary end point. ${ }^{15}$ The study demonstrated that manual thrombectomy significantly improved both MBG and STR and reduced final infarct size (as assessed by MRI). In addition, manual thrombectomy was associated with reduced cardiac morality and MACE at 24 month followup. ${ }^{15}$ This case also showed that manual thrombectomy via transradial approach following acute infero-posterior STEMI had better post procedural MBG \& better STR as well as improved in-hospital clinical outcome.

In conclusion, manual thrombectomy may be an effective and safe option for the treatment of very high thrombotic lesions in patients with acute STEMI.

\section{References:}

1. Levine GN, Bates ER, Blankenship JC, Bailey SR, Bittl JA, Cercek B, et al. $2011 \mathrm{ACCF} / \mathrm{AHA} / \mathrm{SCAI}$ guideline for percutaneous coronary intervention: executive summary: a report of the American college of cardiology foundation/ american heart association task force on practice guidelines and the society for cardiovascular angiography and interventions. Circulation 2011;124:2574-609.

2. Keeley EC, Boura JA, Grines CL. Primary angioplasty versus intravenous thrombolytic therapy for acute myocardial infarction: a quantitative review of 23 randomised trials. Lancet 2003;361(9351):13-20.

3. Rezkalla SH, Kloner RA. Coronary no-reflow phenomenon: from the experimental laboratory to the cardiac catheterization laboratory. Catheterization and cardiovascular interventions 2008;72(7):950-7.

4. Sakai K, Inoue K, Nobuyoshi M. Aspiration thrombectomy of a massive thrombotic embolus in acute myocardial infarction caused by coronary embolism. Int Heart $J$ 2007;48:387-92.

5. Libby P. Current concepts of the pathogenesis of the acute coronary syndromes. Circulation 2001;104:365-72.
6. Silber S, Albertsson P, Aviles FF, Camici PG, Colombo A, Hamm $\mathrm{C}$, et al. Task force for percutaneous coronary interventions of the European Society of Cardiology. Guidelines for percutaneous coronary interventions: the task force for percutaneous coronary interventions of the European Society of Cardiology. Eur Heart $J$ 2005;26:804-47.

7. Costantini CO, Stone GW, Mehran R, Aymong E, Grines CL, Cox DA, et al. Frequency, correlates, and clinical implications of myocardial perfusion after primary angioplasty \& stenting with or without glycoprotein IIb/IIIa inhibition, in acute myocardial infarction. J Am Coll Cardiol 2004; 44(2):305-12.

8. Kushner FG, Hand M, Smith SC, Jr., King SB, 3rd, Anderson JL, Antman EM, et al. 2009 focused updates: ACC/AHA guidelines for the management of patients with ST-elevation myocardial infarction (updating the 2004 guideline and 2007 focused update) and ACC/AHA/SCAI guidelines on percutaneous coronary intervention (updating the 2005 guideline and 2007 focused update) a report of the American College of Cardiology Foundation/American Heart Association Task Force on Practice Guidelines. J Am Coll Cardiol 2009;54(23):2205-41.

9. Van de Werf F, Bax J, Betriu A, Blomstrom-Lundqvist C, Crea F, Falk V, et al. Management of acute myocardial infarction in patients presenting with persistent ST-segment elevation: the Task Force on the Management of ST-Segment Elevation Acute Myocardial Infarction of the European Society of Cardiology. Eur Heart $J$ 2008;29(23):2909-45.

10. Burzotta F, Trani C, Romagnoli E, Mazzari MA, Rebuzzi AG, De Vita $\mathbf{M}$, et al. Manual thrombus-aspiration improves myocardial reperfusion: the randomized evaluation of the effect of mechanical reduction of distal embolization by thrombus-aspiration in primary and rescue angioplasty (REMEDIA) trial. J Am Coll Cardiol 2005;46(2):371-6.

11. De Luca L, Sardella G, Davidson CJ, De Persio G, Beraldi M, Tommasone $\mathrm{T}$, et al. Impact of intracoronary aspiration thrombectomy during primary angioplasty on left ventricular remodelling in patients with anterior ST elevation myocardial infarction. Heart 2006; 92(7):951-57.

12. Mongeon FP, Belisle P, Joseph L, Eisenberg MJ, Rinfret S. Adjunctive thrombectomy for acute myocardial infarction: A bayesian meta-analysis. Circulation 2007;3(1):6-16.

13. Svilaas T, Vlaar PJ, van der Horst IC, Diercks GF, de Smet BJ, van den Heuvel AF, et al. Thrombus aspiration during primary percutaneous coronary intervention. The New England Journal of Medicine 2008;358(6):557-67.

14. Vlaar PJ, Svilaas T, van der Horst IC, Diercks GF, Fokkema ML, de Smet BJ, et al. Cardiac death and reinfarction after 1 year in the Thrombus Aspiration during Percutaneous coronary intervention in Acute myocardial infarction Study (TAPAS): a 1-year follow-up study. Lancet 2008;371(9628):1915-20.

15. Sardella G, Mancone M, Canali E, Di Roma A, Benedetti G, Stio R, et al. Impact of thrombectomy with EXPort Catheter in Infarct-Related Artery during Primary Percutaneous Coronary Intervention (EXPIRA Trial) on cardiac death. The American Journal of Cardiology 2010;106(5):624-29. 